\title{
The Effect of Three Different Impression Techniques on the Treatment Stress Changes for Operator
}

ISSN: 2637-7764

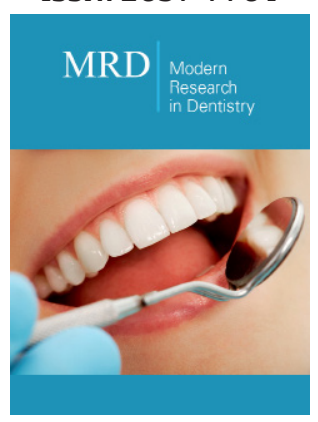

*Corresponding author: Akikazu Shinya, DDS, PhD, Department of Dental Materials Science, School of Life Dentistry at Tokyo, The Nippon Dental University, 1-9-20 Fujimi, Chiyoda-ku, Tokyo 102-8158, Japan

Submission: 觜 February 08, 2020

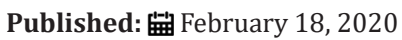

Volume 4 - Issue 5

How to cite this article: Akikazu Shinya, Sakura Shiratori, Akinori Niitsuma, Minori Hatta. The Effect of Three Different Impression Techniques on the Treatment Stress Changes for Operator. Mod Res Dent. 4(5). MRD.000600.2020.

DOI: $10.31031 /$ MRD.2020.04.000600

Copyright@ Akikazu Shinya, This article is distributed under the terms of the Creative Commons Attribution 4.0 International License, which permits unrestricted use and redistribution provided that the original author and source are credited.

\author{
Akikazu Shinya ${ }^{1,2 *}$, Sakura Shiratori ${ }^{3}$, Akinori Niitsuma ${ }^{3}$ and Minori Hatta ${ }^{3}$ \\ ${ }^{1}$ Department of Dental Materials Science, School of Life Dentistry at Tokyo, The Nippon Dental \\ University, Tokyo, Japan \\ ${ }^{2}$ Department of Biomaterials Science and Turku Clinical Biomaterials Centre (TCBC), Institute \\ of Dentistry, University of Turku, Finland
}

${ }^{3}$ Department of Crown and Bridge, School of Life Dentistry at Tokyo, The Nippon Dental University, Tokyo, Japan

\begin{abstract}
s
The aim of this study was to evaluate the changes in the dentist's stress caused by taking an impression, using either the optical impression technique based on digital technology or two different conventional impression techniques. The study was performed following the principles outlined in the Declaration of Helsinki on experimentation involving human subjects, and the protocol was reviewed and approved by the Ethics Committee. Three different impression techniques were used in this study.

1: Agar-alginate combined impression technique (Hydrocolloid impression).

2: Silicone single impression technique (Silicone impression).

3: Optical impression technique (optical impression).

Based on these results, it can be concluded that optical impression is a lower stress technique for the operator, and more time efficient.
\end{abstract}

Keywords: Intraoral scanner; Digital impression; Stress; Impression techniques

\section{Introduction}

The digital technology has brought many benefits to the clinic in the form of digital dentistry. In this field, optical impression taking is regarded as the most impressive technology. Optical impressions offer an easy and effective procedure that is friendly to both patient and dentist and allows for fast transfer of impression data between the dental clinic and the laboratory [1]. As the indirect technique is the most common method for the fabrication of dental restorations, taking impressions is one of the most common procedures in clinical dentistry. An accurate impression is essential for accurate manufacturing of dental restorations. Given this situation, the impression technique might be burden not only for the patient, but also for the dentist. The mental and/or physical stress caused by impression taking might lead to impaired concentration and reduced quality of clinical work. This means that this stress may lead to problems for the patient to the patient. The aim of this study was to evaluate the changes in the dentist's stress caused by taking an impression, using either the optical impression technique based on digital technology or two different conventional impression techniques. The null hypothesis was that there would be no difference in the stress changes in operators between the three different impression techniques.

\section{Methods}

The study population consisted of clinical trainee (dentists) as subjects experienced with impressions and undergraduate dental student (students) as subjects inexperienced with impressions. The study protocol was described in detail to potential subjects, and they were fully informed about the possible risks and benefits. Those who signed an informed consent form were included in the study. Twelve subjects (seven dentists and five students) who fulfilled the following criteria were recruited: good general and mental health, nonsmoking. The study was performed following the principles outlined in the Declaration of Helsinki on experimentation involving human subjects, and the protocol was reviewed and 
approved by the Ethics Committee of the Nippon Dental University (NDU-T2016-05). A left maxillary first premolar which was to be restored with a CAD/CAM resin composite crown was selected as the impression target.

The impression was taken from patient-simulating humanoid robot, in order to have the test condition would simulate the clinical situation as closely as possible. Three different impression techniques were used in this study.

1: Agar-alginate combined impression technique (Hydrocolloid impression, Acelloid combine with Algiace II, Dentsply Sirona, York, PA, USA).

2: Silicone single impression technique (Silicone impression, Tosicon pastel, Dentsply Sirona, York, PA, USA).

3: Optical impression technique (optical impression, CEREC Omnicam, Dentsply Sirona, York, PA, USA).
The subjects had nothing eat or drink for 1 hour and sat quietly for 15 minutes before taking the impression. Saliva was collected by placing a collecting paper under the tongue for 30 seconds. Salivary amylase activity (SAA) was measured in an automatic salivary amylase activity monitor (NIPRO, Osaka, Japan), by attaching the collecting paper in contact with the reagent paper [2,3]. Subjects took impressions three times in one day, and SAA was measured before and after the first and third impressions (Figure 1). The total numbers of impressions were fifteen, taken over five consecutive days. The treatment time was measured as the time between beginning the taking of the impression and its completion. The value of the stress change $(\mathrm{kU} / \mathrm{L})$ was calculated by subtracting the SAA value before the impression from that afterwards [4]. Statistical analysis was performed as follows. The Kruskal-Wallis test followed by the Mann-Whitney U test was used to compare differences between impression methods. Treatment time and stress changes were analyzed by one-way ANOVA and Tukey's honestly significant difference test with significance level of 0.05 .

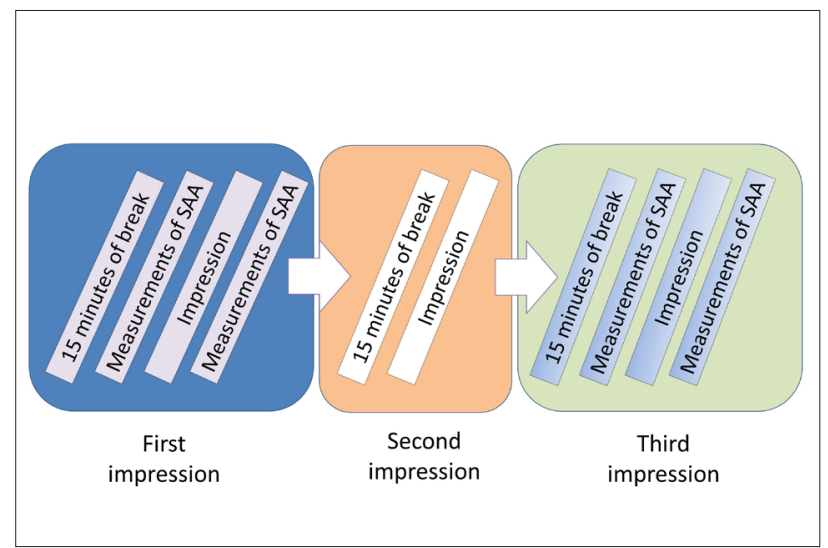

Figure 1: Impression schedule.

\section{Result}

The results of the stress changes for all impression techniques are presented in Figure 2. Optical impression shows a significantly smaller stress change $(\mathrm{p}<0.05)$ compared to the two conventional impression techniques. There is no significant difference between the two conventional impression techniques. The results of the treatment times for all impression techniques are presented in Figure 3 . The difference between treatment times was statistically significant $(\mathrm{p}<0.05)$, and optical impression treatment time was significantly shorter. There was no significant difference between the two conventional impression techniques. The mean treatment times of the impression techniques were as follows: Hydrocolloid impression, $190.56 \pm 31.67 \mathrm{~s}$; silicone impression, $246.54 \pm 42.86 \mathrm{~s}$; optical impression, $43.32 \pm 13.13$ s. Figure $4 \mathrm{a}$-f shows the results of the stress changes for all test groups. There were no significant differences between any of these conditions.

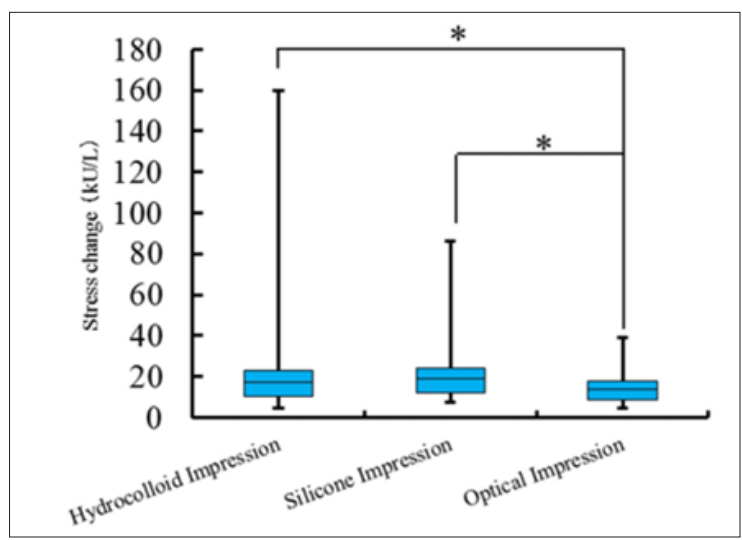

Figure 2: Stress change for all impression technique. 


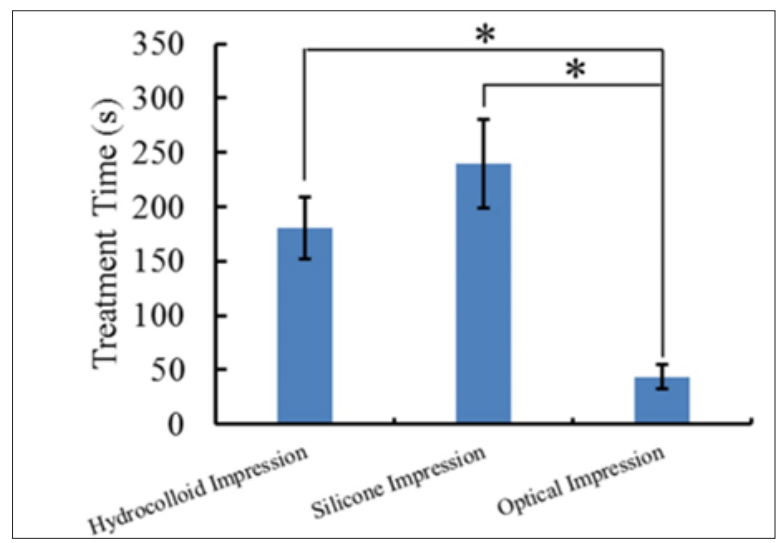

Figure 3: Treatment time.

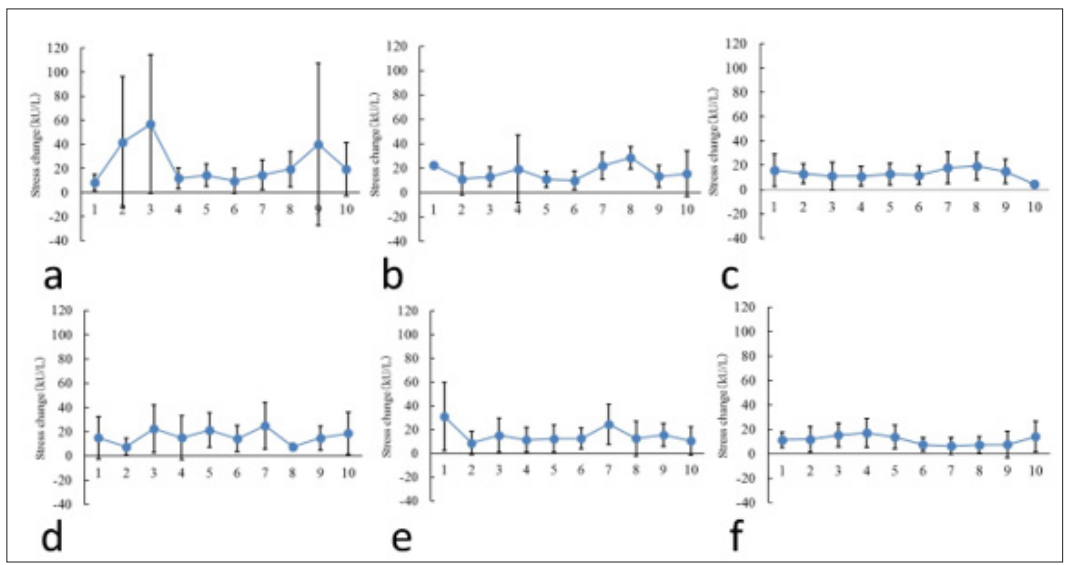

Figure 4: Stress changes for all groups

a: Students-Hydrocolloid impression.

b: Students-Silicone impression.

c: Students-Optical impression.

d: Dentists-Hydrocolloid impression.

e: Dentists-Silicone impression.

f: Dentists-Optical impression.

\section{Discussion}

It is possible that the small stress changes seen for optical impressions are because it is easy to see the situation of the impression while it is still imposing, it is not time consuming, it is easy to start the procedure over even if it is already in progress, and there are no necessary physical procedures. On the other hand, for hydrocolloid impression and silicon impression, the operator may feel uncomfortable because it is impossible to check how the impression is going before removing the impression material. Of the procedures compared in this study, the hydrocolloid and silicone impression methods are physical methods, while the optical impression is a digital method involving no impression materials. Thus, the operator cannot avoid physical processes requiring skill by using the optical camera, but also get the results in significantly shorter time. It is not unreasonable to suppose that this reduces stress.

\section{Conclusion}

Within the limitations of this study, the following conclusions can be drawn

1. The optical impression showed significantly lower less stress changes than the two conventional impression techniques. Thus, the null hypothesis was rejected.

2. The overall treatment time for optical impression was also significantly shorter than those for the two conventional impression techniques.

3. There were no significant differences between the two different levels of experience for the three different impression techniques.

Based on these results, it can be concluded that optical impression is a lower stress technique for the operator, and more time efficient. 


\section{References}

1. Murai Y, Saitoh M, Minowa E, Islam ST, Kobashi M, et al. (2019) Evaluation of stress in a comparison between the digital impression and conventional impression for children. JJADD 9(1): 1-7.

2. Yamaguchi M, Kanemaru M, Kanemori T, Mizuno Y (2003) Flowinjection-type biosensor system for salivary amylase activity. Biosens Bioelectron 18(5-6): 835-840.
3. Yamaguchi M, Kanemori T, Kanemaru M, Takai N, Mizuno Y, et al. (2004) Performance evaluation of salivary amylase activity monitor. Biosensors and Bioelectronics 20(3): 491-497.

4. Shetty V, Zigler C, Robles TF, Elashoff D, Yamaguchi M (2011) Developmental validation of a point-of-care, salivary $\alpha$-amylase biosens. Psychoneuroendocrinology 36(2): 193-199.

For possible submissions Click below: 